\title{
Generalization of Quasi Convex Functions using Convolution
}

\author{
Khalida Inayat Noor ${ }^{1}$, Samar Abbas ${ }^{2,}{ }^{*}$ and Bushra Kanwal ${ }^{3}$ \\ ${ }^{1}$ Mathematics Department, COMSATS University, Park Road, Islamabad, Pakistan \\ e-mail: khalidanoor@hotmail.com; khalidan@gmail.com \\ ${ }^{2}$ Mathematics Department, COMSATS University, Park Road, Islamabad, Pakistan \\ e-mail: samarabbax@gmail.com \\ ${ }^{3}$ Mathematics Department, COMSATS University, Park Road, Islamabad, Pakistan \\ e-mail: bushrakanwal27pk@gmail.com
}

\begin{abstract}
In this paper, an up-to-date generalization of the class $\mathrm{C}^{\star}$ of quasi-convex functions is given by introducing new class $C_{\mathfrak{g}}^{\star}[\mathfrak{a}, \mathfrak{b}]$. Furthermore its basic properties, its relationship with other subclasses of $\mathbf{S}$, inclusion relations and some other interesting properties are derived.
\end{abstract}

\section{Introduction}

Let $\mathrm{A}$ denote the class of analytic functions in the open unit disk $\mathrm{E}=\{z:|z|<1\}$ defined by the power series

$$
\ell(z)=z+\sum_{\kappa=2}^{\infty} a_{\kappa} z^{\kappa}
$$

The convolution or Hadamard product of two analytic functions

$$
\ell(z)=\sum_{\kappa=0}^{\infty} a_{\kappa} z^{\kappa+1}, \jmath(z)=\sum_{\kappa=0}^{\infty} b_{\kappa} z^{\kappa+1}, z \in \mathrm{E}, \kappa=0,1,2, \cdots
$$

Received: July 24, 2020; Revised \& Accepted: August 16, 2020

2010 Mathematics Subject Classification: 30C45, 30C50.

Keywords and phrases: analytic functions, open unit disk, quasi convex function, convolution, Janowski type functions.

${ }^{*}$ Corresponding author 
is defined as

$$
(\ell * \jmath)(z)=\sum_{\kappa=0}^{\infty} a_{\kappa} b_{\kappa} z^{\kappa+1} .
$$

Moreover, we say that $\ell(z)$ is subordinate to $\jmath(z)$ written as $\ell(z) \prec \jmath(z)$ if there exists a function $\omega(z)$ analytic in $\mathrm{E}$ with $\omega(0)=0$ and $|\omega(z)|<1$, for all $z \in \mathrm{E}$, such that $\ell(z)=\jmath(\omega(z))$.

Let $\mathrm{S}, \mathrm{C}, \mathrm{S}^{\star}, \mathrm{K}$ and $\mathrm{C}^{\star}$ be the sub-classes of $\mathrm{A}$, which contain univalent, convex, starlike, close-to-convex and quasi-convex functions respectively. For several interesting geometric properties and deatils of these classes, one can refer to the standard books [4, 12]. It is well known [4] that $\ell(z)$ defined in (1.1) is convex if and only if $z \ell^{\prime}(z) \in \mathrm{S}^{\star}$ and that $\ell(z)$ is quasi convex if and only if $z \ell^{\prime}(z)$ is close to convex, see [11, 10].

Let $\mathcal{H}$ be the class of functions

$$
p(z)=1+\sum_{\kappa=1}^{\infty} c_{\kappa} z^{\kappa},
$$

that are regular in $\mathrm{E}$ with $p(0)=1$. Then $p \in \mathrm{P}[\mathfrak{a}, \mathfrak{b}],-1 \leq \mathfrak{b}<\mathfrak{a} \leq 1$ if and only if $p(z) \prec \frac{1+\mathfrak{a} z}{1+\mathfrak{b} z}$, or equivalently

$$
p(z)=\frac{(\mathfrak{a}+1) h(z)-(\mathfrak{a}-1)}{(\mathfrak{b}+1) h(z)-(\mathfrak{b}-1)}
$$

where $h \in \mathrm{P}[1,-1]=\mathrm{P}$, the class of functions with positive real part. Also $p \in \mathrm{P}(\gamma)$ if and only if

$$
\Re\{p(z)\}>\gamma, \quad 0<\gamma<1 .
$$

First time Janowski [6] considered and studied extensively this class of functions. The classes $\mathrm{C}[\mathfrak{a}, \mathfrak{b}], \mathrm{S}^{\star}[\mathfrak{a}, \mathfrak{b}], \mathrm{K}[\mathfrak{a}, \mathfrak{b}]$ and $\mathrm{C}^{\star}[\mathfrak{a}, \mathfrak{b}]$ were defined and discussed in [1, 13].

Let $\ell(z) \in \mathrm{A}$. Denote by $\mathrm{Ð}^{\sigma}: \mathrm{A} \longrightarrow \mathrm{A}$, the operator defined by

$$
\bigoplus^{\sigma} \ell(z)=\frac{z}{(1-z)^{(\sigma+1)}} * \ell(z)=z+\sum_{\kappa=2}^{\infty} \digamma_{\kappa}(\sigma) a_{\kappa} z^{\kappa}, \quad(\sigma>-1),
$$

with

$$
\digamma_{\kappa}(\sigma)=\frac{(\sigma+1)_{\kappa-1}}{(\kappa-1) !}
$$


where $(\xi)_{\kappa}$ is Pochhammer symbol given as

$$
(\xi)_{\kappa}= \begin{cases}1, & \text { for } \kappa=0 \\ \xi(\xi+1)(\xi+2) \cdots(\xi+\kappa-1), & \text { for } \kappa \in \mathbb{N} .\end{cases}
$$

It is obvious that $\mathrm{Ð}^{0} \ell(z)=\ell(z), \mathrm{Ð}^{1} \ell(z)=z \ell^{\prime}(z)$ and

$$
\mathrm{Ð}^{n} \ell(z)=\frac{z\left(z^{n-1} \ell(z)\right)^{n}}{n !}, \forall n=0,1,2,3 \cdots .
$$

The operator $\mathrm{Ð}^{\sigma} \ell(z)$ is called Ruscheweyh derivative of $\ell(z)$, see [15].

Definition 1. Let $\ell$ and $\mathfrak{g} \in \mathrm{A}$, such that $(\mathfrak{g} * \ell) \neq 0$ in $\mathrm{E}$. Then $\ell$ is said to belong to $S_{\mathfrak{g}}^{\star}[\mathfrak{a}, \mathfrak{b}]$ if and only if

$$
\frac{z(\mathfrak{g} * \ell)^{\prime}(z)}{(\mathfrak{g} * \ell)(z)} \in \mathrm{P}[\mathfrak{a}, \mathfrak{b}] .
$$

We note that if $\mathfrak{g}=\frac{z}{(1-z)}$, then $\mathrm{S}_{\mathfrak{g}}^{\star}[\mathfrak{a}, \mathfrak{b}]=\mathrm{S}^{\star}[\mathfrak{a}, \mathfrak{b}]$.

Definition 2. Let $\ell \in \mathrm{A},(\mathfrak{g} * \ell)^{\prime} \neq 0$ in $\mathrm{E}$ for $\mathfrak{g} \in \mathrm{A}$. Then $\ell$ is said to belong to $C_{\mathfrak{g}}[\mathfrak{a}, \mathfrak{b}]$ if and only if

$$
\frac{\left(z(\mathfrak{g} * \ell)^{\prime}(z)\right)^{\prime}}{(\mathfrak{g} * \ell)^{\prime}(z)} \in \mathrm{P}[\mathfrak{a}, \mathfrak{b}] .
$$

Note that if $\mathfrak{g}=\frac{z}{(1-z)}$, then $\mathrm{C}_{\mathfrak{g}}[\mathfrak{a}, \mathfrak{b}]=\mathrm{C}[\mathfrak{a}, \mathfrak{b}]$.

Definition 3. Let $\ell \in A$. Then $\ell \in K_{\mathfrak{g}}[\mathfrak{a}, \mathfrak{b}]$ if and only if there exists $\mathfrak{u} \in \mathrm{S}_{\mathfrak{g}}^{\star}[\mathfrak{a}, \mathfrak{b}]$ with $(\mathfrak{g} * \mathfrak{u}) \neq 0$ such that

$$
\frac{z(\mathfrak{g} * \ell)^{\prime}(z)}{(\mathfrak{g} * \mathfrak{u})(z)} \in \mathrm{P}[\mathfrak{a}, \mathfrak{b}], \quad z \in \mathrm{E} .
$$

Note that if $\mathfrak{g}=\frac{z}{(1-z)}$, then $\mathrm{K}_{\mathfrak{g}}[\mathfrak{a}, \mathfrak{b}]=\mathrm{K}[\mathfrak{a}, \mathfrak{b}]$.

Definition 4. Let $\ell \in A$. Then $\ell \in \mathrm{C}_{\mathfrak{g}}^{\star}[\mathfrak{a}, \mathfrak{b}]$ if and only if there exists $\mathfrak{u} \in \mathrm{C}_{\mathfrak{g}}[\mathfrak{a}, \mathfrak{b}]$ with $(\mathfrak{g} * \mathfrak{u}) \neq 0$ such that

$$
\frac{\left(z(\mathfrak{g} * \ell)^{\prime}\right)^{\prime}(z)}{(\mathfrak{g} * \mathfrak{u})^{\prime}(z)} \in \mathrm{P}[\mathfrak{a}, \mathfrak{b}], \quad z \in \mathrm{E} .
$$

If $\mathfrak{g}=\frac{z}{(1-z)}, \Longrightarrow \mathrm{C}_{\mathfrak{g}}^{\star}[\mathfrak{a}, \mathfrak{b}]=\mathrm{C}^{\star}[\mathfrak{a}, \mathfrak{b}]$. 
Now we have $\ell \in \mathrm{C}_{\mathfrak{g}}^{\star}[\mathfrak{a}, \mathfrak{b}, \sigma] \Longleftrightarrow \mathrm{Ð}^{\sigma} \ell \in \mathrm{C}_{\mathfrak{g}}^{\star}[\mathfrak{a}, \mathfrak{b}]$, where $\bigoplus^{\sigma}$ is the Ruscheweyh derivative operator [15].

Definition 5. Let $\ell \in \mathrm{A}$ and $\mathfrak{g} \in \mathrm{A}$. Then $\ell \in \mathrm{Q}_{\mathfrak{g}}[\mathfrak{a}, \mathfrak{b} ; \alpha], 0 \leq \alpha<1$ if and only if there exists $\mathfrak{u} \in \mathrm{C}_{\mathfrak{g}}[\mathfrak{a}, \mathfrak{b}]$ with $(\mathfrak{g} * \mathfrak{u}) \neq 0$ such that

$$
(1-\alpha) \frac{(\mathfrak{g} * \ell)^{\prime}}{(\mathfrak{g} * \mathfrak{u})^{\prime}}+\alpha \frac{\left(z(\mathfrak{g} * \ell)^{\prime}\right)^{\prime}}{(\mathfrak{g} * \mathfrak{u})^{\prime}} \in \mathrm{P}[\mathfrak{a}, \mathfrak{b}], \quad z \in \mathrm{E} .
$$

We note that

$$
\begin{aligned}
& Q_{\mathfrak{g}}[\mathfrak{a}, \mathfrak{b} ; \mathfrak{o}]=K_{\mathfrak{g}}[\mathfrak{a}, \mathfrak{b}] \\
& Q_{\mathfrak{g}}[\mathfrak{a}, \mathfrak{b} ; \mathfrak{l}]=C_{\mathfrak{g}}^{\star}[\mathfrak{a}, \mathfrak{b}] .
\end{aligned}
$$

\section{Some Preliminary Results}

Lemma 2.1. 14] $\psi \in \mathrm{C}, \mathfrak{g} \in \mathrm{S}^{\star}$ and $F$ is analytic in $\mathrm{E}$ with $F(0)=1$, then

$$
\frac{\psi * F \mathfrak{g}}{\psi * \mathfrak{g}} \subset \bar{C} o F(\mathrm{E})
$$

where $\bar{C}$ o is the closed convex hull.

Lemma 2.2. [8] Let $u=u_{1}+\iota u_{2}$ and $v=v_{1}+\iota v_{2}$, and let $\Psi$ be the set of functions $\Psi(u, v)$ satisfying:

(a) $\Psi(u, v)$ is continuous in a domain $D$ of $\mathbb{C} \times \mathbb{C}$.

(b) $(1,0) \in D$ and $\Re \Psi(1,0)>0$.

(c) $\Re \Psi\left(\iota u_{2}, v_{1}\right) \leq 0$ when $\left(\iota u_{2}, v_{1}\right) \in D$ and $v \leq-\frac{1}{2}\left(1+u_{2}^{2}\right)$.

If $p(z)$, given by (1.4), is an analytic function in $\mathrm{E}$ such that $\left(p(z), z p^{\prime}(z)\right) \in D$ and $\Re \Psi\left(p(z), z p^{\prime}(z)\right)>0$, for $z \in \mathrm{E}$, then $\Re p(z)>0$.

Lemma 2.3. [7] If $\aleph$ and $\Lambda$ are regular in $\mathrm{E}, \aleph(0)=\Lambda(0)=0$, $\aleph$ maps $\mathrm{E}$ onto a many sheeted region which is starlike with respect to origin, and $\frac{\aleph^{\prime}}{\Lambda^{\prime}} \in \mathrm{P}$, then $\stackrel{\aleph}{\Lambda} \in \mathrm{P}$.

Lemma 2.4. [16] For a real number $\sigma(\sigma>0)$, we have

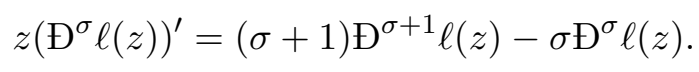




\section{Main Results}

We present our main work in this section.

Theorem 3.1. Let $\ell \in \mathrm{S}^{\star}[\mathfrak{a}, \mathfrak{b}]$ and $\mathfrak{g} \in$ C. Then $\ell \in \mathrm{S}_{\mathfrak{g}}^{\star}[\mathfrak{a}, \mathfrak{b}]$.

Proof. Consider

$$
\begin{aligned}
\frac{z(\mathfrak{g} * \ell)^{\prime}}{(\mathfrak{g} * \ell)} & =\frac{\left(\mathfrak{g} * z \ell^{\prime}\right)}{(\mathfrak{g} * \ell)} \\
& =\frac{\mathfrak{g} * \frac{z \ell^{\prime}}{\ell} \ell}{(\mathfrak{g} * \ell)} \\
& =\frac{\mathfrak{g} * F \ell}{(\mathfrak{g} * \ell)} \subset \bar{C} o F(\mathrm{E}) .
\end{aligned}
$$

Since $F \in \mathrm{P}[\mathfrak{a}, \mathfrak{b}]$ and $\mathfrak{g} \in \mathrm{C}$, we use Lemma 2.1 to have the required result that $\ell \in \mathrm{S}_{\mathfrak{g}}^{\star}[\mathfrak{a}, \mathfrak{b}]$.

Theorem 3.2. The class $\mathrm{S}_{\mathfrak{g}}^{\star}[\mathfrak{a}, \mathfrak{b}]$ is closed under convolution with convex functions.

Proof. Let $\ell \in \mathrm{S}_{\mathfrak{g}}^{\star}[\mathfrak{a}, \mathfrak{b}]$. Then

$$
\frac{z(\mathfrak{g} * \ell)^{\prime}}{(\mathfrak{g} * \ell)}=p(z) \in \mathrm{P}[\mathfrak{a}, \mathfrak{b}] .
$$

Let $\psi \in \mathrm{C}$, we have

$$
\begin{aligned}
\frac{z[\mathfrak{g} *(\psi * \ell)]^{\prime}}{[\mathfrak{g} *(\psi * \ell)]} & =\frac{\psi * z(\mathfrak{g} * \ell)^{\prime}}{\psi *(\mathfrak{g} * \ell)} \\
& =\frac{\psi * \frac{z(\mathfrak{g} * \ell)^{\prime}}{(\mathfrak{g} * \ell)}(\mathfrak{g} * \ell)}{\psi *(\mathfrak{g} * \ell)} \\
& =\frac{\psi * p(\mathfrak{g} * \ell)}{\psi *(\mathfrak{g} * \ell)} \in \bar{C} \operatorname{oo}(\mathrm{E}) .
\end{aligned}
$$

Since $\ell \in \mathbf{S}_{\mathfrak{g}}^{\star}[\mathfrak{a}, \mathfrak{b}]$, it follows that $p \in \mathrm{P}[\mathfrak{a}, \mathfrak{b}]$.

$$
(\mathfrak{g} * \ell) \in \mathrm{S}^{\star}[\mathfrak{a}, \mathfrak{b}] \subset \mathrm{S}^{\star} .
$$

We use Lemma 2.1 to conclude that $\frac{\psi * p(\mathfrak{g} * \ell)}{\psi *(\mathfrak{g} * \ell)}$ lies in the convex hull of $p(\mathrm{E})$ and therefore $\psi * \ell \in \mathbf{S}_{\mathfrak{g}}^{\star}[\mathfrak{a}, \mathfrak{b}]$. 
Theorem 3.3. Let $\ell \in \mathrm{C}_{\mathfrak{g}}^{\star}[\mathfrak{a}, \mathfrak{b}]$ with respect to $\mathfrak{u} \in \mathrm{C}_{\mathfrak{g}}[\mathfrak{a}, \mathfrak{b}]$ and let $\psi \in \mathrm{C}$. Then $\psi * \ell \in \mathrm{C}_{\mathfrak{g}}^{\star}[\mathfrak{a}, \mathfrak{b}]$ with respect to $z(\mathfrak{g} * \mathfrak{u})^{\prime}=\mathfrak{u}_{1}$.

Proof. By definition $C_{\mathfrak{g}}^{\star}[\mathfrak{a}, \mathfrak{b}]$ implies $\mathfrak{g} * \mathfrak{u} \in \mathrm{C}[\mathfrak{a}, \mathfrak{b}]$.

Let

$$
p(z)=\frac{\left(z(\mathfrak{g} * \ell)^{\prime}\right)^{\prime}}{(\mathfrak{g} * \mathfrak{u})^{\prime}} .
$$

Then $p \in \mathrm{P}[\mathfrak{a}, \mathfrak{b}]$ in E. Also, it is known [4] that $\mathrm{C}[\mathfrak{a}, \mathfrak{b}] \subset \mathrm{C} \subset \mathrm{S}^{\star}$.

Now,

$$
\begin{aligned}
\frac{\left(z(\mathfrak{g} *(\psi * \ell))^{\prime}\right)^{\prime}}{(\mathfrak{g} * \psi * \mathfrak{u})^{\prime}} & =\frac{z\left(\mathfrak{g} * z(\psi * \ell)^{\prime}\right)^{\prime}}{\psi * z(\mathfrak{g} * \mathfrak{u})^{\prime}} \\
& =\frac{\psi * \frac{\left(z(\mathfrak{g} * \ell)^{\prime}\right)^{\prime}}{(\mathfrak{g} * \mathfrak{u})^{\prime}} z(\mathfrak{g} * \mathfrak{u})^{\prime}}{\psi * z(\mathfrak{g} * \mathfrak{u})^{\prime}} \\
& =\frac{\psi * p z(\mathfrak{g} * \mathfrak{u})^{\prime}}{\psi * z(\mathfrak{g} * \mathfrak{u})^{\prime}} \\
& =\frac{\psi * p \mathfrak{u}_{1}}{\psi * \mathfrak{u}_{1}} \subset \bar{C} o p(\mathrm{E}) .
\end{aligned}
$$

Since $\psi \in \mathrm{C}$ and $\mathfrak{u}_{\perp} \in \mathrm{S}^{\star}$, the required result follows using Lemma 2.1. That is $(\psi * \ell) \in \mathbf{C}_{\mathfrak{g}}^{\star}[\mathfrak{a}, \mathfrak{b}]$.

Corollary 3.3.1. If $\mathfrak{g}=\frac{z}{(1-z)}$, then Theorem 3.3 leads that $(\psi * \ell) \in \mathrm{C}_{\mathfrak{g}}^{\star}[\mathfrak{a}, \mathfrak{b}]=$ $\mathrm{C}^{\star}[\mathfrak{a}, \mathfrak{b}]$.

Theorem 3.4. $C_{\mathfrak{g}}[\mathfrak{a}, \mathfrak{b}] \subset S_{\mathfrak{g}}^{\star}[\mathfrak{a}, \mathfrak{b}]$.

Proof. Consider

$$
\frac{z(\mathfrak{g} * \ell)^{\prime}}{(\mathfrak{g} * \ell)}=p(z)
$$

Let $\ell \in \mathrm{C}_{\mathfrak{g}}[\mathfrak{a}, \mathfrak{b}]$, it can be simply seen that $p$ is analytic and $p(0)=1$ for $z \in \mathrm{E}$.

By logarithmically differentiation, we have

$$
\left[1+\frac{(\mathfrak{g} * \ell)^{\prime \prime}}{(\mathfrak{g} * \ell)^{\prime}}\right]=p(z)+\frac{z p^{\prime}(z)}{p(z)} \in \mathrm{P}[\mathfrak{a}, \mathfrak{b}] .
$$

Since $\ell \in \mathrm{C}_{\mathfrak{g}}[\mathfrak{a}, \mathfrak{b}]$. Now using Lemma 2.2 it follows that $p \in \mathrm{P}[\mathfrak{a}, \mathfrak{b}]$ which implies $\ell \in \mathrm{S}_{\mathfrak{g}}^{\star}[\mathfrak{a}, \mathfrak{b}]$. 
Corollary 3.4.1. If $\mathfrak{g}=\frac{z}{(1-z)}$, then Theorem 3.4 follows that $\mathrm{C}[\mathfrak{a}, \mathfrak{b}] \subset \mathrm{S}^{\star}[\mathfrak{a}, \mathfrak{b}]$.

Theorem 3.5. Let $0<\alpha<1$. Then

$$
\mathrm{Q}_{\mathfrak{g}}[\mathfrak{a}, \mathfrak{b} ; \alpha] \subset \mathrm{K}_{\mathfrak{g}}[\mathfrak{a}, \mathfrak{b}] .
$$

Proof. From Definition 5 , it follows that $\ell \in Q_{\mathfrak{g}}[\mathfrak{a}, \mathfrak{b}]$ if and only if

$$
£(z)=(1-\alpha) \ell(z)+\alpha z \ell^{\prime}(z) \in \mathrm{K}_{\mathfrak{g}}[\mathfrak{a}, \mathfrak{b}] .
$$

We can express $(3.2)$ as

$$
\begin{gathered}
\ell(z)=\frac{1}{\alpha} z^{\left(1-\frac{1}{\alpha}\right)} \int_{0}^{z} \tau^{\left(\frac{1}{\alpha}-2\right)} £(\tau) d \tau, £ \in \mathrm{K}_{\mathfrak{g}}[\mathfrak{a}, \mathfrak{b}] \\
=\frac{c+1}{z^{c}} \int_{0}^{z} \tau^{(c-1)} £(\tau) d \tau ; \quad\left(\frac{1}{\alpha}=c+1\right) \\
=\left(\sum_{\kappa=1}^{\infty} \frac{c+1}{c+\kappa} z^{\kappa}\right) * £(z), \quad \kappa=1,2,3, \cdots \\
\ell(z)=\left(\phi_{c} * £\right)(z),
\end{gathered}
$$

where $\sum_{\kappa=1}^{\infty} \frac{c+1}{c+\kappa} z^{\kappa}$ is convex, see [1] and $£ \in \mathrm{K}_{\mathfrak{g}}[\mathfrak{a}, \mathfrak{b}]$ is preserved under convex convolution. In fact from Theorem 3.2, $\phi_{c} * \ell_{1} \in \mathrm{S}_{\mathfrak{g}}^{\star}[\mathfrak{a}, \mathfrak{b}]$ if $\ell_{1} \in \mathrm{S}_{\mathfrak{g}}^{\star}[\mathfrak{a}, \mathfrak{b}]$, and

$$
\begin{aligned}
\frac{z\left(\phi_{c} * \mathfrak{g} * £\right)^{\prime}}{\phi_{c} *\left(\mathfrak{g} * \ell_{\mathfrak{1}}\right)} & =\frac{z\left(\mathfrak{g} *\left(\phi_{c} * £\right)\right)^{\prime}}{\mathfrak{g} *\left(\phi_{c} * \ell_{1}\right)} . \\
& =\frac{z(\mathfrak{g} * \ell)^{\prime}}{\mathfrak{g} * £_{1}},
\end{aligned}
$$

where $\left(\phi_{c} * \ell_{1}\right)=£_{1} \in \mathrm{S}_{\mathfrak{g}}^{\star}[\mathfrak{a}, \mathfrak{b}]$, by Theorem 3.2 and $\ell$ is given by equation (3.2), consequently $\ell \in \mathrm{K}_{\mathfrak{g}}[\mathfrak{a}, \mathfrak{b}]$ in $\mathrm{E}$.

Corollary 3.5.1. Let $\mathfrak{g}=\frac{z}{(1-z)}$, it follows from Theorem 3.5 that $\mathrm{Q}[\mathfrak{a}, \mathfrak{b} ; \alpha] \subset \mathrm{K}[\mathfrak{a}, \mathfrak{b}]$, for $0<\alpha<1$.

Theorem 3.6. Let $\mathfrak{g} \in \mathrm{S}^{\star}[\mathfrak{a}, \mathfrak{b}],-1 \leq \mathfrak{b}<\mathfrak{a} \leq 1$ and let $\frac{z \ell^{\prime}}{\mathfrak{g}} \in \mathrm{P}(\beta)$ for $z \in \mathrm{E}$. Then $\Re \frac{\left(z \ell^{\prime}\right)^{\prime}}{\mathfrak{g}^{\prime}}>\beta, 0 \leq \beta<1$, for $|z|<r_{1}$ where $r_{1}$ is the least positive root in $(0,1)$ of the equation

$$
1-(\mathfrak{a}+2) r+(2 \mathfrak{b}-1) r^{2}+\mathfrak{a} r^{3}=0 .
$$


Proof. For $z \in \mathrm{E}$, we have

$$
\frac{z \ell^{\prime}}{\mathfrak{g}}=h
$$

where $\mathfrak{g} \in \mathbf{S}^{\star}[\mathfrak{a}, \mathfrak{b}]$ and $h \in \mathrm{P}(\beta)$.

Differentiating logarithmically, we have

$$
\frac{\left(z \ell^{\prime}\right)^{\prime}}{\mathfrak{g}^{\prime}}=h+\frac{\mathfrak{g}}{\mathfrak{g}^{\prime}} h^{\prime}
$$

it follows that

$$
\Re\left(\frac{\left(z \ell^{\prime}\right)^{\prime}}{\mathfrak{g}^{\prime}}-\beta\right) \geq \Re\left(|h-\beta|-\left|\frac{\mathfrak{g}}{\mathfrak{g}^{\prime}} \| h^{\prime}\right|\right) .
$$

Using distortion results, that are given in [4] as

$$
\begin{gathered}
\left|\frac{\mathfrak{g}}{\mathfrak{g}^{\prime}}\right| \leq \frac{r(1-\mathfrak{b} r)}{1-\mathfrak{a} r} \\
\left|h^{\prime}\right| \leq \frac{2 \Re[h-\beta]}{1-r^{2}} .
\end{gathered}
$$

Using these results in equation (3.5), we have

$$
\Re\left[\frac{\left(z \ell^{\prime}\right)^{\prime}}{\mathfrak{g}^{\prime}}-\beta\right] \geq \frac{\Re(h-\beta)\left[1-(\mathfrak{a}+2) r+(2 \mathfrak{b}-1) r^{2}+\mathfrak{a} r^{3}\right]}{(1-\mathfrak{a} r)\left(1-r^{2}\right)} .
$$

The right hand side of equation (3.8) is positive for $|z|=r<r_{1}$, where $r_{1}$ is the least positive root of (3.4), which completes our proof.

Corollary 3.6.1. As a special case, when $\mathfrak{a}=0, \mathfrak{b}=-1$ and $\beta=0$ we have $\Re \frac{z \ell^{\prime}}{\mathfrak{g}}>0, \mathfrak{g} \in \mathrm{S}^{\star}\left(\frac{1}{2}\right)$ for $z \in \mathrm{E}$. In this case $\Re \frac{\left(z \ell^{\prime}\right)^{\prime}}{\mathfrak{g}^{\prime}}>0$ for $|z|<\frac{1}{3}$.

Theorem 3.7. Let $\ell \in \mathrm{S}_{\mathfrak{g}}^{\star}[\mathfrak{a}, \mathfrak{b}]$. Then $\ell \in \mathrm{C}_{\mathfrak{g}}[\mathfrak{a}, \mathfrak{b}]$ for $|z|<r_{o}=2-\sqrt{3}$.

Proof. Since $\ell \in \mathbf{S}_{\mathfrak{g}}^{\star}[\mathfrak{a}, \mathfrak{b}]$, therefore we can write

$$
\frac{z(\mathfrak{g} * \ell)^{\prime}}{(\mathfrak{g} * \ell)}=h
$$


where $h$ is a Carathéodory function $h \in \mathrm{P}$, this implies $\frac{z \ell_{1}{ }^{\prime}}{\ell_{1}}=h$, where $\ell_{1}=\mathfrak{g} * \ell$. Differentiating logarithmically, we have

$$
\frac{z \ell_{1}^{\prime}}{\ell_{1}}=h+\frac{z h^{\prime}}{h} .
$$

Using distortion results for the class $\mathrm{P}$, see [3, 4, 5]. We have

$$
\begin{aligned}
\Re \frac{z(\mathfrak{g} * \ell)^{\prime}}{(\mathfrak{g} * \ell)}=\Re \frac{z \ell_{\mathfrak{1}}^{\prime}}{\ell_{1}} & \geq \frac{1-r}{1+r}-\frac{2 r}{1-r^{2}} \\
& =\frac{1-4 r+r^{2}}{1-r^{2}},
\end{aligned}
$$

by using mean value theorem and with some computations we get

$$
T(r)=2-\sqrt{3} \in(0,1), \quad T(r)=2-\sqrt{3} \in(0,1) .
$$

Thus

$$
\Re \frac{z(\mathfrak{g} * \ell)^{\prime}}{(\mathfrak{g} * \ell)} \geq \frac{1-4 r+r^{2}}{1-r^{2}}>0, \quad \text { for } r<r_{o}=2-\sqrt{3}
$$

Corollary 3.7.1. Let $\mathfrak{g}=\frac{z}{(1-z)}$, so Theorem 3.7 follows that if $\ell \in \mathrm{S}^{\star}[\mathfrak{a}, \mathfrak{b}]$, then $\ell \in \mathrm{C}[\mathfrak{a}, \mathfrak{b}]$ for $|z|<r_{o}=2-\sqrt{3}$.

A converse case of Theorem 3.5, we have following result

Theorem 3.8. Let $\ell \in \mathrm{K}_{\mathfrak{g}}[\mathfrak{a}, \mathfrak{b}]$. Then $\ell \in \mathrm{Q}_{\mathfrak{g}}[\mathfrak{a}, \mathfrak{b} ; \alpha]$ for

$$
|z|<r_{\alpha}=\frac{1}{2 \alpha+\sqrt{4 \alpha^{2}-2 \alpha+1}} .
$$

Proof. Consider $\ell \in \mathrm{K}_{\mathfrak{g}}[\mathfrak{a}, \mathfrak{b}]$. Then we can write

$$
(1-\alpha) \ell+\alpha z \ell^{\prime}=\phi_{c} * \ell,
$$

where

$$
\phi_{c}(z)=\sum_{\kappa=1}^{\infty}[(\kappa-1) \alpha+1] z^{\kappa} .
$$


It can be verified with simple computations that $\phi_{c}(z) \in \mathrm{C}$ for $|z|<r_{\alpha}$, where $r_{\alpha}$ is given by $(3.9)$. Then, using Remark 1 that the class $\mathrm{K}_{\mathfrak{g}}[\mathfrak{a}, \mathfrak{b}]$ is preserved under convex convolution, it follows that $\ell \in Q_{\mathfrak{g}}[\mathfrak{a}, \mathfrak{b} ; \alpha]$ for $|z|<r_{\alpha}, r_{\alpha}$ is given by 3.9 .

Corollary 3.8.1. For $\mathfrak{g}=\frac{z}{(1-z)}$, Theorem 3.8 follows that if $\ell \in \mathrm{K}[\mathfrak{a}, \mathfrak{b}]$, then $\ell \in \mathbf{Q}[\mathfrak{a}, \mathfrak{b} ; \alpha]$, where as $|z|<r_{\alpha}$ and $r_{\alpha}$ is given by (3.9).

Theorem 3.9. If $z \ell^{\prime} \in \mathrm{K}_{\mathfrak{g}}[\mathfrak{a}, \mathfrak{b}]$, then $\ell \in \mathrm{C}_{\mathfrak{g}}^{\star}[\mathfrak{a}, \mathfrak{b}]$.

Proof. Consider $\ell \in \mathrm{C}_{\mathfrak{g}}^{\star}[\mathfrak{a}, \mathfrak{b}]$ and defined by (1.8), so

$$
\Longrightarrow \frac{\left(\left(\mathfrak{g} * z \ell^{\prime}\right)\right)^{\prime}}{(\mathfrak{g} * \mathfrak{u})^{\prime}} \in \mathrm{P}[\mathfrak{a}, \mathfrak{b}] .
$$

Let $F=z \ell^{\prime}$,

$$
\Longrightarrow \frac{(\mathfrak{g} * F)^{\prime}}{(\mathfrak{g} * \mathfrak{u})^{\prime}} \in \mathrm{P}[\mathfrak{a}, \mathfrak{b}] .
$$

This shows that $F \in \mathrm{K}_{\mathfrak{g}}[\mathfrak{a}, \mathfrak{b}]$. Using Alexander type relation between $\mathrm{C}$ and $\mathrm{S}^{\star}$ we get

$$
\frac{z(\mathfrak{g} * F)^{\prime}}{(\mathfrak{g} * G)} \in \mathrm{P}[\mathfrak{a}, \mathfrak{b}]
$$

where $G=z \mathfrak{u}^{\prime}$. This completes our proof.

Remark 1. Using Theorem 3.9 and alike techniques of Theorem 3.3 , we can easily prove that the class $K_{\mathfrak{g}}[\mathfrak{a}, \mathfrak{b}]$ is preserved under convex convolution that is, if $\ell \in \mathrm{K}_{\mathfrak{g}}[\mathfrak{a}, \mathfrak{b}]$ and $\phi \in \mathrm{C}$, then $\phi * \ell \in \mathrm{K}_{\mathfrak{g}}[\mathfrak{a}, \mathfrak{b}]$ in $\mathrm{E}$.

\section{Some Inclusion Relations}

Remark 2. $C_{\mathfrak{g}}^{\star}[\mathfrak{a}, \mathfrak{b}] \not \subset S_{\mathfrak{g}}^{\star}[\mathfrak{a}, \mathfrak{b}]$ and $S_{\mathfrak{g}}^{\star}[\mathfrak{a}, \mathfrak{b}] \not \subset C_{\mathfrak{g}}^{\star}[\mathfrak{a}, \mathfrak{b}]$.

Proof. Noor proved that the class $\mathrm{C}^{\star}$ does not properly contained in the class $\mathrm{S}^{\star}$ and $\mathrm{S}^{\star} \not \subset \mathrm{C}^{\star}$, see [11]. If $\mathfrak{g}=\frac{z}{(1-z)}$, then our result is obvious. 
Theorem 3.10. $C_{\mathfrak{g}}^{\star}[\mathfrak{a}, \mathfrak{b}] * \mathrm{~S}_{\mathfrak{g}}^{\star}[\mathfrak{a}, \mathfrak{b}] \subset \mathrm{K}_{\mathfrak{g}}[\mathfrak{a}, \mathfrak{b}]$.

Proof. Let $\ell \in \mathrm{C}_{\mathfrak{g}}^{\star}[\mathfrak{a}, \mathfrak{b}]$ and $\mathfrak{u} \in \mathrm{S}_{\mathfrak{g}}^{\star}[\mathfrak{a}, \mathfrak{b}]$.

Consider that

$$
\begin{aligned}
(\ell * \mathfrak{u}) & =\left(\ell * z \mathfrak{u}^{\prime}\right), \quad \text { where } \mathfrak{u} \in C_{\mathfrak{g}}[\mathfrak{a}, \mathfrak{b}] . \\
& =z(\ell * \mathfrak{u})^{\prime} \\
& =z \ell^{\prime} * \mathfrak{u} .
\end{aligned}
$$

It follows from Alexander type relations that $\ell * \mathfrak{u} \in \mathrm{K}_{\mathfrak{g}}[\mathfrak{a}, \mathfrak{b}]$.

Next theorem is given as a special case of Lemma 2.3 .

Theorem 3.11. $C_{\mathfrak{g}}^{\star}[\mathfrak{a}, \mathfrak{b}] \subset K_{\mathfrak{g}}[\mathfrak{a}, \mathfrak{b}]$.

Proof. Let $\aleph(z)=z(\mathfrak{g} * \ell)^{\prime}(z)$ and $\Lambda(z)=(\mathfrak{g} * \mathfrak{u})(z)$ be analytic functions in $\mathrm{E}$ with $\aleph(0)=\Lambda(0)=0$ and $\Lambda \in \mathrm{S}^{\star}$. Then Lemma 2.3 implies

$$
\frac{\aleph^{\prime}}{\Lambda^{\prime}} \in \mathrm{P} \Longrightarrow \frac{\aleph}{\Lambda} \in \mathrm{P}, \quad \text { for } z \in \mathrm{E},
$$

which leads to our required result.

Theorem 3.12. Let $\sigma \geq 1$. Then

$$
\mathrm{C}_{\mathfrak{g}}^{\star}[\sigma+1, \mathfrak{a}, \mathfrak{b}] \subset \mathrm{C}_{\mathfrak{g}}^{\star}[\sigma, \mathfrak{a}, \mathfrak{b}] .
$$

Proof. For $\ell \in$ A. Using Lemma 2.4 we can easily derive the identity

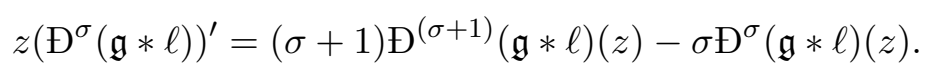

Set

$$
\frac{\left(z\left(Ð^{\sigma}(\mathfrak{g} * \ell)\right)^{\prime}\right)^{\prime}(z)}{\left(\bigoplus^{\sigma}(\mathfrak{g} * \mathfrak{u})\right)^{\prime}(z)}=h(z) .
$$

Then $h(z)$ is analytic in E with $h(0)=1$. We shall show that

$$
\Re h(z)>0, \quad z \in \mathrm{E} .
$$


First we show that

$$
\mathrm{C}_{\mathfrak{g}}[\sigma+1, \mathfrak{a}, \mathfrak{b}] \subset \mathrm{C}_{\mathfrak{g}}[\sigma, \mathfrak{a}, \mathfrak{b}]
$$

For this, let $\mathfrak{u} \in \mathrm{C}_{\mathfrak{g}}[\sigma+1, \mathfrak{a}, \mathfrak{b}]$ and set

$$
\frac{\left(z\left(\bigoplus^{\sigma}(\mathfrak{g} * \mathfrak{u})\right)^{\prime}\right)^{\prime}(z)}{\left(\bigoplus^{\sigma}(\mathfrak{g} * \mathfrak{u})\right)^{\prime}(z)}=H_{o}(z)
$$

Then $H_{o}(z)$ is analytic and $H_{o}(0)=1$. Using identity $(3.10)$ for $\mathfrak{u}$ together with 3.12 we have

$$
\frac{(\sigma+1)\left(\bigoplus^{\sigma+1}(\mathfrak{g} * \mathfrak{u})\right)^{\prime}(z)}{\left(\bigoplus^{\sigma}(\mathfrak{g} * \mathfrak{u})\right)^{\prime}(z)}=H_{o}(z)+\sigma .
$$

Differentiating both sides of 3.13 logarithmically and using 3.12 , with some computations we obtain

$$
\frac{\left(z\left(\boxplus^{\sigma+1}(\mathfrak{g} * \mathfrak{u})\right)^{\prime}(z)\right.}{\left(\bigoplus^{\sigma+1}(\mathfrak{g} * \mathfrak{u})\right)^{\prime}(z)}=H_{o}(z)+\frac{z H_{o}^{\prime}(z)}{H_{o}(z)+\sigma} .
$$

Since $\mathfrak{u} \in \mathrm{C}_{\mathfrak{g}}[\sigma+1, \mathfrak{a}, \mathfrak{b}]$, therefore right hand of (3.14) belongs to $\mathrm{P}[\mathfrak{a}, \mathfrak{b}]$. From (3.12), 3.14 and a well-known Lemma 2.2 due to Miller [8, also see [9], it follows that $\Re H_{o}(z) \in \mathrm{P}[\mathfrak{a}, \mathfrak{b}]$ in $\mathrm{E}$. This proves that $\mathfrak{u} \in \mathrm{C}[\sigma, \mathfrak{a}, \mathfrak{b}]$.

Now with similar procedure and from (3.11), we get

$$
\frac{\left(z\left(\boxplus^{\sigma+1}(\mathfrak{g} * \ell)\right)^{\prime}\right)^{\prime}(z)}{\left(\bigoplus^{\sigma+1}(\mathfrak{g} * \mathfrak{u})\right)^{\prime}(z)}=h(z)+\frac{z h^{\prime}(z)}{h_{o}(z)+\sigma} .
$$

Again applying Lemma 2.2, we obtain from 3.15 that $\Re h(z) \in \mathrm{P}[\mathfrak{a}, \mathfrak{b}]$ in E, which proves that $\ell \in \mathrm{C}_{\mathfrak{g}}^{\star}[\sigma, \mathfrak{a}, \mathfrak{b}]$ in $\mathrm{E}$. This establishes our required inclusion result.

Theorem 3.13. The class $C_{\mathfrak{g}}^{\star}[\mathfrak{a}, \mathfrak{b}]$ is preserved under the following integral operators defined in [2, 7]

(a)

$$
\begin{aligned}
\ell_{1}(z) & =\int_{0}^{z} \frac{\ell(\tau)}{\tau} d \tau \\
& =\left(\mathfrak{g}_{1} * \ell\right)(z), \quad\left(\mathfrak{g}_{1}\right)(z)=-\log (1-z) .
\end{aligned}
$$


(b)

$$
\begin{aligned}
\ell_{2}(z) & =\frac{2}{z} \int_{0}^{z} \frac{\ell(\tau)}{\tau} d \tau \\
& =\left(\mathfrak{g}_{2} * \ell\right)(z), \quad\left(\mathfrak{g}_{2}\right)(z)=-2[z+\log (1-z)] .
\end{aligned}
$$

(c)

$$
\begin{aligned}
\ell_{3}(z) & =\frac{1+\varsigma}{z^{\varsigma}} \int_{0}^{z} \tau^{\varsigma-1} \ell(\tau) d \tau \\
& =\left(\mathfrak{g}_{3} * \ell\right)(z), \quad\left(\mathfrak{g}_{3}\right)(z)=\sum_{\sigma+1}^{\infty} \frac{1+\varsigma}{\sigma+\varsigma} z^{\sigma}, \quad \text { where } \sigma \in \mathbb{N} .
\end{aligned}
$$

It can be easily seen that $\mathfrak{g}_{1}, \mathfrak{g}_{2} \in \mathrm{C}$. In [15], Ruscheweyh showed that $\mathfrak{g}_{3} \in \mathrm{C}$ in $\mathrm{E}$. Consequently using Theorem 3.3 , it follows that $\ell_{\mathfrak{i}} \in \mathrm{C}_{\mathfrak{g}}^{\star}[\mathfrak{a}, \mathfrak{b}]$, where $\mathfrak{i}=1,2,3$.

\section{References}

[1] O. Altintaş and Ö. Ö. Kiliç, Coefficient estimates for a class containing quasi-convex functions, Turk. J. Math. 42 (2018), 2819-2825.

https://doi.org/10.3906/mat-1805-90

[2] S. M. Barnardi, Convex and starlike univalent functions, Trans. Amer. Math. Soc. 135 (1969), 429-446. https://doi.org/10.1090/S0002-9947-1969-0232920-2

[3] P. L. Duren, Univalent Functions, Springer-Verlag, Berlin, 1983.

[4] A. W. Goodman, Univalent Functions, Vols. I and II, Polygonal Publishing House, Washington, NJ, 1983.

[5] W. K. Hayman, Multivalent Functions, Cambridge University Press, U.K., 1967.

[6] W. Janowski, Some extremal problems for certain families of analytic functions I, Ann. Polon. Math. 28 (1973), 297-326.

https://doi.org/10.4064/ap-28-3-297-326

[7] R. J. Libera, Some classes of regular univalent functions, Proc. Amer. Math. Soc. 16 (1965), 755-758. https://doi.org/10.1090/S0002-9939-1965-0178131-2 
[8] S. S. Miller, Differential Inequalities and Carathéodory functions, Bull. Amer. Math. Soc. 81 (1975), 79-81. https://doi.org/10.1090/S0002-9904-1975-13643-3

[9] S. S. Miller and P. T. Mocanu, Differential Subordination: Theory and Applications, Vol. 225, Marcel Dekker Inc., New York, Basel, 2000. https://doi.org/10.1201/9781482289817

[10] K. I. Noor and D. K. Thomas, On quasi convex univalent functions, Inter. J. Math. Math. Sci. 3 (1980), 255-266. https://doi.org/10.1155/S016117128000018X

[11] K. I. Noor, On quasi convex functions and related topics I, J. Math. Math. Sci. 10(2) (1987), 241-258. https://doi.org/10.1155/S0161171287000310

[12] Ch. Pommerenke, Univalent Functions, Vandenhoeck and Ruprecht, Gottingen, 1975 .

[13] M. S. Robertson, On the theory of univalent functions, Ann. of Math. 28 (1936), 297-326.

[14] S. Ruscheweyh and T. Sheil-Small, Hadamard products of Schlicht functions and the Pólya-Schoenberg conjecture, Comment. Math. Helv. 48 (1973), 119-135. https://doi.org/10.1007/BF02566116

[15] S. Ruscheweyh, New criteria for univalent functions, Proc. Amer. Math. Soc. 49 (1975), 109-115. https://doi.org/10.1090/S0002-9939-1975-0367176-1

[16] K. Sakaguchi and S. Fukui, An extension of a theorem of S. Ruscheweyh, Bull. Fac. Edu. Wakayama Univ. Nat. Sci. 29 (1980), 1-3.

This is an open access article distributed under the terms of the Creative Commons Attribution License (http://creativecommons.org/licenses/by/4.0/), which permits unrestricted, use, distribution and reproduction in any medium, or format for any purpose, even commercially provided the work is properly cited. 